\title{
Itinéraires Itinéraires
}

Littérature, textes, cultures

2013-1 | 2013

La fiction aujourd'hui

\section{La vérité et la fiction}

ce qu'en disent quelques personnages

\section{Cécile De Bary}

\section{(2) OpenEdition}

Journals

\section{Édition électronique}

URL : http://journals.openedition.org/itineraires/870

DOI : 10.4000/itineraires.870

ISSN : 2427-920X

Éditeur

Pléiade

\section{Édition imprimée}

Date de publication : 1 octobre 2013

Pagination : 167-181

ISBN : 978-2-343-01791-4

ISSN : $2100-1340$

Référence électronique

Cécile De Bary, "La vérité et la fiction », Itinéraires [En ligne], 2013-1 | 2013, mis en ligne le 01 octobre 2013, consulté le 30 avril 2019. URL : http://journals.openedition.org/itineraires/870 ; DOI : 10.4000/ itineraires.870

\section{(c) (i) (9)}

Itinéraires est mis à disposition selon les termes de la licence Creative Commons Attribution - Pas d'Utilisation Commerciale - Pas de Modification 4.0 International. 


\section{La vérité et la fiction \\ Ce qu'en disent quelques personnages}

\section{Résumé}

Faut-il croire les romans qui, dans des passages métatextuels, affirment une confusion entre réalité et fiction? Certainement, si comme dans La Théorie des nuages, d'Audeguy, il s'agit de désigner la fictionnalisation des « îlots référentiels » du récit. Peut-être, s'il est question de signaler la persistance utopique d'un espoir, assimilé au rêve, dans Ourania de Le Clézio. Sans doute, quand, dans La Vérité sur Marie, le narrateur revendique la fictionnalité de son discours, devant l'impossibilité de dire le vrai : il réaménage dès lors explicitement la réalité. Les romans actuels jouent de la fiction et de son extension, avec conscience.

Mots clés : fictionnalisation, imaginaire, référence fictionnelle, utopie, métatextuel

\section{Abstract}

Should we believe novels that, through metatextual passages, confound reality with fiction? Probably, when it means pointing out the fictionalization of referential elements of narrative, as in Audeguy's La Théorie des nuages. Perhaps, when it involves identifying the utopian persistence of hope within dreams, as in Le Clezio's Ourania. Undoubtedly, when the narrator of La Vérité sur Marie assumes his use of fiction, as it is impossible to tell the truth, and consequently explicitly rearranges reality. Current novels use fiction and the extension of it, and willfully so.

Keywords: fictionalization, imaginary, fictional reference, utopia, metatextual

Dans plusieurs romans de la première décennie du nouveau millénaire, se trouve énoncé une sorte de pacte d'indécidabilité : des personnages expliquent ou pensent qu'ils ne font pas de différence entre l'imaginaire et le réel, entre ce qui est inventé et ce qui ne l'est pas. Quoique pris eux-mêmes dans la fiction, puisque émis par des personnages, ces discours semblent constituer des pistes de lecture, au point qu'on peut se demander s'il s'agit dans ces romans d'inciter le lecteur à mettre en doute le partage entre le fictif et le référentiel. 
Dans La Théorie des nuages ${ }^{1}$, Virginie Latour, bibliothécaire détachée auprès du couturier Akira Kumo, écoute les histoires qu'il lui raconte. Cela permet à Stéphane Audeguy, l'auteur du livre, de raconter au lecteur ces mêmes histoires. Ce dispositif narratif pourrait se rapprocher d'une double énonciation si cet échange, rappelé en quelques brefs passages, souvent en début de chapitre, ne passait régulièrement au second plan. La narration des différentes aventures des passionnés de nuages, dont il est question dans ce livre, s'effectue sans guillemets ni décrochage typographique, avec un caractère de discours rapporté qui n'est rappelé que par quelques incises minimales, par surcroît répétitives : le verbe « dire » revient à plusieurs reprises, l'expression « dit Akira Kumo à Virginie Latour » étant répétée deux fois, p. 49 et 98. Parallèlement, la rencontre de Virginie Latour et d'Akira Kumo constitue le fil narratif essentiel du livre, ainsi que ses répercussions sur leurs destins respectifs. Or, sa narration ne diffère guère de celle rattachée à Akira Kumo, que ce soit par le style ou par les procédés narratifs. Quoi qu'il en soit, ce pseudo-discours rapporté permet de faire de Virginie une narrataire, et donc une image du lecteur, et ce même si elle devient à son tour narratrice auprès d'Akira Kumo, à la fin du livre (lui racontant les aventures d'un certain Abercrombie). Or, vers le début du livre, Virginie Latour comprend des histoires de Kumo qu' « on ne peut pas dire qu'elles soient fausses, on ne peut pas dire qu'il les fabrique de toutes pièces; mais il est évident qu'il les aménage, qu'il y ajoute des éléments. [...] Virginie Latour a réfléchi à tout ça; elle a conclu que ça n'avait pas d'importance ${ }^{2}$ ». Il semblerait que le lecteur soit invité à faire de même, et à écouter ces histoires « avec un plaisir sans mélange », comme l'énonce la suite du passage.

Le narrateur d'Ourania, de J.-M.G. Le Clézio, rencontre au Mexique Raphaël Zacharie, qui lui parle de Campos, la communauté dont il fait partie. Il remarque :

" Je te raconte cela, mais tu sais, pour nous autres, à Campos, ça n'est qu'une histoire. » J'avais l'air surpris, il a ajouté : «Une histoire, tu sais, un conte qu'on raconte aux enfants pour les endormir, ou aux vieux pour qu'ils se souviennent de leur jeunesse. »

J'ai dit: «Alors, tout ce que tu me racontes est inventé? » Il s'est mis à rire. «Inventé, ou vrai, pour nous à Campos ça veut dire la même chose. Nous ne considérons pas comme vrai uniquement ce que nous touchons ou ce que nous voyons. Les choses mortes continuent d'exister, elles changent, elles ne sont plus les mêmes quand elles sont sur le bout de notre langue ${ }^{3}$. »

Il ne s'agit a priori que de croyances propres à Campos. Si l'on retrouve en ce passage un récit dans le récit, et donc une forme de double énonciation,

1. Stéphane Audeguy, La Théorie des nuages [2005], Paris, Gallimard, coll. « Folio », 2007.

2. Ibid., p. 79-80.

3. J.-M.G. Le Clézio, Ourania [2006], Paris, Gallimard, coll. « Folio », 2007, p. 37. 
le narrateur premier, Daniel Sillitoe, marque une distance par rapport à ces propos. Cependant, il les prolonge par la suite en reliant de plusieurs manières Campos à Ourania, un pays qu'il a imaginé lorsqu'il était enfant. Ainsi, il revoit Raphaël à la sortie d'une conférence qu'il a donnée, et celui-ci lui annonce qu'il va lui raconter son histoire. Il remarque alors : «Je crois que c'est ce soir-là que j'ai pensé pour la première fois à Ourania, au pays que j'avais inventé dans mon enfance ${ }^{4}$. » De manière plus nette encore, le fondateur de Campos est appelé « l'homme qui a créé la véritable Ourania ${ }^{5} \gg$. Enfin, vingt-cinq ans après l'expropriation de cette dernière, dont il a été témoin avec Dahlia, une femme qu'il aimait, Daniel Sillitoe remarque que «ce qui nous unit encore, Dahlia et moi, ce qui nous permet d'espérer, c'est la certitude que le pays d'Ourania a vraiment existé, d'en avoir été les témoins ${ }^{6} »$. Ce pays imaginaire qui a vraiment existé ressemble fort à ce que raconte Raphaël...

Dans le roman de Jean-Philippe Toussaint La Vérité sur Marie, le narrateur, séparé de la femme qu'il aime, tente de reconstituer ce qui lui est arrivé en son absence, en particulier lors du malaise cardiaque de son amant, un certain Jean-Christophe de G. Vers la fin du roman, il remarque :

Je savais qu'il y avait sans doute une réalité objective des faits - ce qui s'est réellement passé cette nuit-là dans l'appartement de la rue de $\mathrm{La}$ Vrillière -, mais que cette réalité me resterait toujours étrangère, je pourrais seulement tourner autour, l'aborder sous différents angles, la contourner et revenir à l'assaut, mais je buterais toujours dessus, comme si ce qui s'était réellement passé cette nuit-là m'était par essence inatteignable, hors de portée de mon imagination et irréductible au langage. J'aurais beau reconstruire cette nuit en images mentales qui auraient la précision du rêve, j'aurais beau l'ensevelir de mots qui auraient une puissance d'évocation diabolique, je savais que je n'atteindrais jamais ce qui avait été pendant quelques instants la vie même, mais il m'apparut alors que je pourrais peut-être atteindre une vérité nouvelle, qui s'inspirerait de ce qui avait été la vie et la transcenderait, sans se soucier de vraisemblance ou de véracité, et ne viserait qu'à la quintessence du réel, sa moelle sensible, vivante et sensuelle, une vérité proche de l'invention, ou jumelle du mensonge, la vérité idéale ${ }^{7}$.

Ces affirmations conduisent à douter de la source du récit. Les lecteurs vont-ils jusqu'à se poser la question de la « vérité », ce qui rejoindrait le titre du livre ${ }^{8}$ ? De fait, ce récit comporte un certain nombre d'incohérences, ou en tout cas des rectificatifs désinvoltes. Ainsi, Jean-Christophe de G. ne

4. Ibid., p. 77.

5. Ibid., p. 293.

6. Ibid., p. 336, c'est la dernière phrase du livre.

7. Jean-Philippe Toussaint, La Vérité sur Marie, Paris, Minuit, 2009, p. 165-166.

8. Un autre passage métatextuel, à contenu semblable, reprend le titre du livre : « Je savais la vérité sur Marie. » (Ibid., p. 74.) 
s'appelle pas finalement Jean-Christophe de G. mais Jean-Baptiste ${ }^{9}$, ce qui n'empêche pas le narrateur de continuer de l'appeler ainsi. Sa nuit d'amour avec Marie, reconstituée par le narrateur lorsqu'il observe qu'elle a bu un verre de grappa avec lui ${ }^{10}$, n'a peut-être pas existé, puisqu'elle lui confie plus tard qu'elle n'était " pas sa maîtresse ${ }^{11} \ldots$ ».

\section{Le pacte fictionnel et l'oubli de la vérité}

Il demeure qu'habituellement, un lecteur ne s'interroge pas sur la vérité du discours qu'il lit, dès lors qu'il est fictionnel. C'est le pacte fictionnel qui le conduit à oublier cette question : en somme, le lecteur de fiction sait que ce qu'il lit est faux. C'est la condition même de sa lecture. Tant que Les Lettres portugaises ont été considérées comme de vraies lettres, elles n'ont pas été lues comme des fictions ${ }^{12}$. De même, penser que Wolfgang Hildesheimer a écrit une véritable biographie avec Marbot, en 1981, c'est méconnaître le statut de cet ouvrage ${ }^{13}$. Le titre de La Vérité sur Marie, désigné comme un « roman » par sa couverture, pourrait donc bien être ironique.

Finalement, la question de la vérité d'un discours se pose seulement quand il prétend désigner la réalité. C'est ainsi que peuvent se comprendre les scrupules de Jean-Jacques Rousseau, en tête de son autobiographie :

J'ai dit le bien et le mal avec la même franchise. Je n'ai rien tu de mauvais, rien ajouté de bon, et s'il m'est arrivé d'employer quelque ornement indifférent, ce n'a jamais été que pour remplir un vide occasionné par mon défaut de mémoire; j'ai pu supposer vrai ce que je savais avoir pu l'être, jamais ce que je savais être faux ${ }^{14}$.

Dès lors, les discours du narrateur de La Vérité sur Marie pourraient s'expliquer par la capacité qu'a la fiction de mimer tout autre discours, en

9. Ibid, p. 66, 69. P. 74, le narrateur admet: « Je me trompais peut-être parfois sur JeanChristophe de G. »

10. Le narrateur explique que cette bouteille est « le détail tangible à partir duquel je pourrais imaginer ce qu'elle avait vécu, qu'à partir de ce détail, de cette seule bouteille de grappa, je pourrais reconstituer tout ce qui s'était passé entre eux dans la chambre » (ibid., p. 51).

11. Ibid., p. 178. Le narrateur s'interroge pourtant: " Qu'avait-elle voulu me dire en me disant qu'elle n'était pas sa maîtresse? » Et il formule différentes hypothèses.

12. Cet ouvrage paru en 1669 ne fut d'abord pas attribué à Guilleragues, qui se présentait comme le traducteur des lettres d'une religieuse portugaise, Marianna Alcofarado, à un officier français. Sur cet exemple et d'autres, voir Christine Montalbetti, «Fiction, réel, référence », Littérature, ${ }^{\circ} 123$, septembre 2001, p. 44-55.

13. Jean-Marie Schaeffer et Dorrit Cohn ont étudié le cas de cette erreur de lecture : «La fiction », dans Jean-Marie Schaeffer, Pourquoi la fiction?, Paris, Le Seuil, coll. « Poétique », 1999, p. 133-230; «Briser le code de la biographie fictionnelle: Sir Andrew Marbot de Wolfgang Hildesheimer », dans Dorrit Cohn, Le Propre de la fiction, Paris, Le Seuil, 2001, p. $125-147$.

14. Second préambule des Confessions de Jean-Jacques Rousseau, édition établie par Alain Grosrichard, Paris, Flammarion, coll. « GF », 2002, p. 29. 
particulier le témoignage. Par cette mimésis formelle, caractéristique du « roman à la première personne ${ }^{15}$ », la fiction prétend restituer le témoignage d'un narrateur épris d'une certaine Marie, retraçant la vie de celle-ci dans la mesure de ce qu'il en sait, et imaginant le reste, un peu à la manière d'un Rousseau, mutatis mutandis. Cette fiction imitant le discours d'un témoin imparfait, relève donc, dans une première analyse, du vraisemblable : l'intervention de la première personne s'opposerait à l'arbitraire de la narration à la troisième personne ${ }^{16}$.

Pour autant, le narrateur de La Vérité sur Marie va plus loin que Rousseau. Dans le passage que j'ai déjà cité, il assume " une vérité proche de l'invention, ou jumelle du mensonge, la vérité idéale ». Le germe d'invention qui était dû chez Rousseau au « défaut de mémoire » enfle chez Toussaint, au point de passer au premier plan. De fait, loin de Marie, le narrateur est un témoin particulièrement défectueux. Plus encore, c'est le rêve qui lui permet de rejoindre Marie.

Je n'avais pas été présent cette nuit-là, mais j'avais accompagné Marie en pensée avec la même intensité émotionnelle que si j'avais été là, comme dans une représentation qui serait advenue sans moi, non pas de laquelle j'aurais été absent, mais à laquelle seuls mes sens auraient participé, comme dans les rêves, où chaque figure n'est qu'une émanation de soi-même, recréée à travers le prisme de notre subjectivité, irradiée de notre sensibilité, de notre intelligence et de nos fantasmes. Même si je ne dormais pas, c'était le mystère irréductible du rêve qui était en train d'agir et de jouer en moi, qui permet à la conscience de construire des images extraordinairement élaborées qui s'agencent dans une succession de séquences apparemment disposées au hasard, avec des ellipses vertigineuses, des lieux qui s'évanouissent et plusieurs personnages de notre vie qui fusionnent, se superposent et se transforment, et qui, malgré cette incohérence radicale, ravivent en nous, avec une intensité brûlante, des souvenirs, des désirs et des craintes, pour susciter, comme rarement dans la vie même, la terreur et l'amour ${ }^{17}$.

Ce passage a une portée métatextuelle évidente, puisque le récit correspond à ce que ce discours énonce du rêve, par sa structure elliptique,

15. Michał Głowiński, «Sur le roman à la première personne », Poétique, no 72, 1987, p. 497-506.

16. «En fait, je pose théoriquement le problème de la troisième personne en littérature. Il ne s'agit pas d'un petit problème. Comment peut-on écrire à la troisième personne en littérature? Une réponse possible est : “On ne peut pas." C'est, d'ailleurs, ma première réponse. En écrivant La Vérité sur Marie, j'ai connu des moments de découragement qui étaient liés au fait que je ne parvenais pas à écrire à la troisième personne. Dans la deuxième partie du livre, Marie est seule et le récit est purement à la troisième personne. Comme Madame Bovary. Mais, au lieu d'être extérieur au récit, le narrateur connaît le personnage. » (Laurent Demoulin, "Entretien avec Jean-Philippe Toussaint», propos recueillis le 26 mai 2009, Culture, le magazine culturel de l'université de Liège, [En ligne], http://culture.ulg.ac.be/ jcms/prod_132357/entretien-avec-jean-philippe-toussaint, consulté le 15 juin 2011.)

17. Jean-Philippe Toussaint, op. cit., p. 167-168. 
concentrée autour de quelques scènes dramatiques autant que dramatisées, une crise cardiaque, la fuite antérieure d'un cheval, un incendie sur l'île d'Elbe. Cette structure étant rapportée à un fonctionnement psychique, il semblerait que le roman dans son ensemble soit destiné à l'éclairer : celui d'un homme épris d'une femme dont il est séparé, et rêvant d'elle avant de la rejoindre effectivement, ce que signale un changement d'énonciation : à la fin du roman, le narrateur s'adresse à Marie, jusque-là objet du récit, en la tutoyant ${ }^{18}$. Marie n'est plus un personnage dont on parle à la troisième personne, elle devient une interlocutrice.

\section{Des personnages qui confondent l'imaginaire et leur réalité}

Chez Stéphane Audeguy, la confusion du réel et de l'imaginaire est également propre au personnage auquel la narration est essentiellement attribuée : "Akira Kumo [...] ne déteste pas mentir, du moment que le mensonge porte une vérité supérieure à celle des faits objectivement constatables ${ }^{19}$. \ C'est de même le narrateur d'Ourania qui a inventé un pays imaginaire dans son enfance et qui cherche les traces de celui-ci au Mexique. C'est encore lui qui assimile Campos à Ourania. Plus généralement, sa narration revient à plusieurs reprises, et dans plusieurs contextes, sur la possibilité du rêve, au sens le plus général du terme.

Marqué par sa mère, qui avait « la conviction que la réalité est un secret, et que c'est en rêvant qu'on est près du monde ${ }^{20} »$, Daniel Sillitoe s'éprend au Mexique de Dahlia, qui réalisera plus tard les projets qu'elle formule auprès de lui, et que sur le moment il disqualifie quelque peu : « Elle rêvait tout haut ${ }^{21}$. » La rencontre avec Lili, une prostituée bientôt disparue, est encore caractérisée par l'onirisme ${ }^{22}$. C'est surtout Campos qui est le lieu du rêve : c'est un rêve réalisé ${ }^{23}$, voire un paradis ${ }^{24}$. Plus ponctuellement, on y enseigne notamment en rêvant ${ }^{25}$.

Les ambiguïtés de l'ouvrage, du fait d'une évaluation ambivalente, ont déjà été signalées ${ }^{26}$. Ces ambiguïtés portent essentiellement sur le rêve,

18. Ibid., p. 205.

19. Stéphane Audeguy, op. cit., p. 42.

20. J.-M. G. Le Clézio, op. cit., p. 13.

21. Ibid., p. 120.

22. Voir ibid., p. 132 : «J'ai glissé dans mon rêve. J'ai laissé Lili, je suis parti sans me retourner. » Voir aussi les pages 208-209 (qui sont plus critiques).

23. Ibid., p. 234 et 251. Dans son exode, encore, la communauté suit un rêve du Conseiller, se dirigeant vers une île dont il a eu la « vision » (p. 240).

24. Voir notamment ibid., p. 235 et 239.

25. Ibid., p. 113.

26. Voir en particulier Claude Cavallero, «L'utopie dans Ourania de J.-M.G. Le Clézio : dilemme du rêve et du réquisitoire ", dans Sylvain Santi, Jean-Pol Madou et Laurent Van Eynde (dir.), Mythe et Création 2, l'œuvre, l'imaginaire, la société, Chambéry, université de Savoie, laboratoire Langages, Littératures, Sociétés, coll. «Écriture et représentation », 
terme qui s'étend à toute capacité d'imagination. Ainsi, l'amour porté à Lili, prostituée énigmatique qui fait l'objet de plusieurs pages extrêmement lyriques, est qualifié de « chimères $^{27}$ ». Les habitants de Campos portent une utopie, puisqu'ils ont « essayé de vivre autrement». Pour autant, ce sont des « rêveurs immatures ${ }^{28}$ » et leur paradis est illusoire ${ }^{29}$. Emporio est une utopie moins radicale, mais son destin parallèle à Campos rapproche les deux expériences ${ }^{30}$. C'est surtout Juan Uacus qui incarne cette dimension utopique, qui apparaît a posteriori comme une illusion, mais digne d'intérêt $^{31}$. Par ailleurs, comme je l'ai écrit, alors que Dahlia semblait rêvasser, elle réalisera ses projets. Si le narrateur a manqué d'amour à son égard, c'est peut-être parce qu'il n'a pas cru à ceux-ci. En tout cas, dans l'ensemble du livre, le rêve est à la fois l'irréalisable et le refus de l'intolérable. L'imagination permet de contester cet intolérable. Dès lors, cet Ourania, qui a « vraiment existé », c'est tout l'espoir que porte l'humanité, malgré l'état du monde, même si cet espoir paraît chimérique ${ }^{32}$.

Cette valorisation de l'imagination se retrouve bien sûr chez Toussaint, de manière beaucoup moins ambiguë. Pour autant, l'imagination chez ce dernier auteur n'est pas utopique, encore moins politique. Elle est une pente de l'amour. Elle permet d'atteindre une " vérité idéale », qui se rapproche de la "vérité supérieure à celle des faits objectivement constatables » d'Audeguy.

Chez Audeguy, c'est surtout le plaisir de raconter (et d'écouter) des histoires qui est mis en avant. Et ce plaisir suscite l'invention, les

2007, p. 209-221. Voir aussi Isa Van Acker, «Ourania : petite victoire sur fond de débris, J.-M.G. Le Clézio, Gallimard, $2006 »$ (note de lecture), Les Cahiers J.-M.G. Le Clézio, nº 1, A propos de Nice, 2008, en particulier p. 174.

27. J.-M. G. Le Clézio, op. cit., p. 267.

28. Ibid., p. 245.

29. Voir ibid., p. 235. Sur la distance entre la description de Raphaël et ce que peut observer le narrateur, voir notamment Marina Salles «Ourania de J.M.G. Le Clézio : une utopie historisée, un roman politique », Itinerários (Araraquara), n 32, janvier 2011, p. 131. Voir aussi Claude Cavallero, op. cit., p. 210-211.

30. Voir notamment J.-M. G. Le Clézio, op. cit., p. 329 : « Il m’a semblé que, d'une certaine façon, il y avait un lien logique entre l'aventure de l'Emporio et celle de Campos. »

31. Le narrateur évoque à son propos : «L'illusion de faire renaître un passé interrompu, de donner un sens à la vie des jeunes garçons et des jeunes filles, leur rendre une fierté, les sortir de l'ornière et les empêcher d'aller se perdre au nord, dans les banlieues de Los Angeles ou de Seattle. » Malgré le terme d'«illusion », les objectifs de Juan Uacus sont évalués positivement (ibid., p. 260).

32. Avant le passage final que j'ai déjà cité, le narrateur fait un bilan de l'état du monde et remarque : «Il n'y a pas de quoi être optimiste. » (Ibid., p. 336.) Voir aussi Claude Cavallero, op. cit., p. 217 : «Campos révèle l'envers de son propre décor. Campos contredit les inégalités socio-économiques du Mexique contemporain dénoncées au fil des pages à travers l'enrichissement indécent des planteurs de fraisiers et des producteurs d'avocats dont chacun sait qu'ils tiennent “toute la Vallée dans leurs mains" » (l'auteur cite Le Clézio, ibid., p. 60). 
" aménagements ». Le narrateur de La Vérité sur Marie cherche quant à lui d'abord à rejoindre la femme qu'il aime, malgré leur séparation, en pensée donc. Une autre différence réside dans l'objet de l'invention. Marie est un personnage de fiction, que le lecteur ne peut connaître par ailleurs. En revanche, Kumo narre les aventures de personnes historiques. Cela pourrait ne pas revenir au même.

\section{Y a-t-il une fictionnalisation des références réelles au sein de la fiction?}

Les théoriciens de la fiction s'opposent entre « intégrationnistes 》 et « ségrégationnistes » : ils s'opposent sur le statut, par exemple de Napoléon dans Guerre et Paix, ou de Paris dans Le Père Goriot. Demeurent-elles, au sein de la fiction, des références réelles ou au contraire ces références se trouvent-elles «fictionnalisées »? En d'autres termes, la question du vrai et du faux peut-elle se poser pour certains énoncés produits par la fiction? John Searle est le premier des ségrégationnistes. Pour lui, les références à des lieux ou des personnes réelles sont des « références réelles » :

\footnotetext{
Le critère qui permet d'établir [ce qui ne relève pas de la fiction] réside dans ce qui compte comme une erreur. [...] si Sherlock Holmes et Watson vont de Baker Street à la gare de Paddington par un itinéraire géographiquement impossible, nous saurons que Conan Doyle a commis une bévue, alors qu'il n'en aurait commise aucune, n'y eût-il jamais de vétéran de la campagne afghane correspondant à la description de John Watson, docteur en médecine ${ }^{33}$.
}

Il ajoute que la fiction peut produire des énoncés sérieux : les « messages » qu'elle transmet, énoncés explicitement par des maximes. Gérard Genette affirme au contraire : «Le texte de fiction ne conduit à aucune réalité extratextuelle, chaque emprunt qu'il fait (constamment) à la réalité $[. .$.$] se transforme en élément de fiction, comme Napoléon dans$ Guerre et Paix ou Rouen dans Madame Bovary ${ }^{34}$. » Dès Figures II, Gérard Genette montre que les maximes de Balzac relèvent d'un vraisemblable artificiel : elles ne sont pas un message que la fiction servirait mais servent au contraire de caution au récit, elles permettent « de rendre compte de la conduite des personnages et de l'enchaînement de ses intrigues » et, inventées de toutes pièces, de le rendre vraisemblable ${ }^{35}$. La manière dont Nabokov détourne dans $A d a$ la maxime qui figure au début d'Anna Karénine, renversant sa signification, fait soupçonner que les énoncés sérieux produits par

33. John R. Searle, «Le statut logique du discours de la fiction », dans Sens et expression, Paris, Minuit, 1979, p. 116.

34. Gérard Genette, Fiction et diction, Paris, Le Seuil, 1991, p. 37.

35. Gérard Genette, «Vraisemblance et motivation », dans Figures II [1969], Paris, Le Seuil, coll. « Points », 1979, p. 79. 
la fiction peuvent être des énoncés pseudo-sérieux, pris dans les logiques de mimésis formelle que nous avons déjà évoquées ${ }^{36}$.

Un argument fort en faveur du ségrégationnisme est le principe d'écart minimal, dégagé par Marie-Laure Ryan, qui établit que nous nous appuyons sur notre univers d'expérience pour reconstituer les univers fictionnels. Tout ce qui n'est pas explicitement présenté comme différent de ce que nous connaissons est semblable. Un bon exemple en est donné par le genre science-fictionnel. Même un personnage d'un texte de ce genre, où tout se trouve a priori éloigné de notre réalité, se déplace comme nous, à moins que le texte ne précise explicitement qu'il use de la téléportation... Sans le principe de l'écart minimal, on ne peut comprendre comment le lecteur peut parvenir à se représenter les univers fictionnels, alors que leur désignation verbale est toujours incomplète ${ }^{37}$. Il en résulte que pour le lecteur, le Paris mentionné par un texte de fiction, c'est Paris. De même, Napoléon, c'est Napoléon, sauf indication contraire ${ }^{38}$. La question des «bévues référentielles » peut donc effectivement se poser. Comme le dit Jean-Marie Schaeffer, « le narrateur d'un roman qui mène son héros dans une forêt de chênes et qui, voulant décrire leur feuillage, décrit en fait un feuillage de hêtres, commet une erreur qui relève de la logique de la référentialité (sauf si des indices nous permettent de construire la figure d'un narrateur "non fiable") ${ }^{39}$ ». Schaeffer " propose de distinguer la question de la fictivité qui concerne le statut de l'acte énonciatif global de la question de la référentialité qui concerne la structure sémantique réalisée, et donc se situe au niveau propositionnel ${ }^{40} »$. Sa remarque portant sur un narrateur « non fiable » montre pourtant que les bévues référentielles sont interprétées en fonction de l'acte énonciatif global.

De fait, Umberto Eco montre que toutes les bévues référentielles ne sont pas susceptibles d'acquérir une égale importance. Dans le chapitre «L'étrange cas de la rue Servandoni » de Six promenades dans les bois $d u$ roman et d'ailleurs, il s'intéresse à une rue mentionnée par Les Trois

36. L'exemple de cette maxime inaugurale d'Anna Karénine et de son détournement par Nabokov est cité par Searle lui-même, op. cit., p. 118.

37. Marie-Laure Ryan, « Reconstructing the Textual Universe: The Principle of Minimal Departure », dans Possible Worlds, Artificial Intelligence and Narrative Theory, Bloomington \& Indianapolis, Indiana University Press, 1991, p. 48-60.

38. «The need for the principle of minimal departure in interpreting fiction is made particularly compelling by historical narratives. If it weren't for the principle, a novel about a character named Napoleon could not convey the feeling that its hero is the Napoleon. » Ibid., p. 52. («La nécessité du principe d'écart minimal pour l'interprétation de la fiction apparaît de manière particulièrement incontestable grâce aux récits historiques. Sans ce principe, un roman portant sur un personnage nommé Napoléon ne pourrait transmettre l'impression que son héros est le Napoléon. » Je traduis.)

39. Jean-Marie Schaeffer, Qu'est-ce qu'un genre littéraire?, Paris, Le Seuil, coll. « Poétique », 1989, p. 84.

40. Ibid. 
Mousquetaires, et qui ne peut pas exister au moment de la diégèse du roman : l'architecte Servandoni, à qui la rue rend hommage, n'était pas encore né. Après avoir reconstitué la géographie de ce quartier à cette époque, il remarque que l'erreur de Dumas n'a en fait pas d'importance pour le lecteur ${ }^{41}$. Il ajoute :

Supposons que, du palais Tréville, Dumas ait fait sortir d'Artagnan rue $\mathrm{du}$ Vieux-Colombier et l'ait fait tourner rue Bonaparte [...]. Ah! non. C'en serait trop. $[\ldots]$

Pourquoi ne pas accepter que d'Artagnan prenne la rue Bonaparte alors que nous l'acceptons pour la rue Servandoni? C'est évident : parce que tout le monde sait qu'il est impossible qu'une rue Bonaparte ait existé dans le Paris du XVII ${ }^{\mathrm{e}}$ siècle, alors que peu de gens le savent pour la rue Servandoni, et la preuve en est que Dumas lui-même l'ignorait.

En ce cas, notre problème ne concerne pas l'ontologie des personnages qui habitent les mondes narratifs, mais le format de l'Encyclopédie du lecteur modèle ${ }^{42}$.

Le problème n'est donc pas un problème de monde fictionnel, et de maintien de la référence réelle en son sein, mais un problème de reconstitution par le lecteur de ce monde. L'encyclopédie dont il dispose lui permet d'effectuer la séparation entre fiction et diction au sein même du texte narratif, et de repérer d'éventuelles erreurs au sein de cette diction ${ }^{43}$. Or, cette réception est modélisée par ce texte, par le « lecteur modèle » qu'il dessine, ainsi que par le «format de l'Encyclopédie » dont il est censé disposer :

Le lecteur modèle prévu par Les Trois Mousquetaires témoigne de la curiosité et du goût pour la reconstitution historique non érudite, il connaît Bonaparte, il a une vague idée de la différence entre les règnes de Louis XIII et Louis XIV; aussi, l'auteur lui fournit une foule d'informations tant au début qu'en cours de récit, et il n'a pas l'intention de se rendre aux Archives nationales pour vérifier si un comte de Rochefort existait vraiment en ce temps-là ${ }^{44}$.

41. L'erreur de Dumas est bien une erreur à l'égard de l'univers de référence. Il faut préciser ce que nous entendons par vrai et faux, puisque Eco remarque qu'on peut dire « il n'est pas vrai que Holmes a une épouse ». En ce cas, la question du vrai et du faux est une question de cohérence interne à l'univers fictionnel, et non une question d'adéquation avec notre univers, question dont je traite ici. (Umberto Eco, «L'étrange cas de la rue Servandoni », dans Six promenades dans les bois du roman et d'ailleurs [1996], Librairie générale française, coll. « Le livre de poche », 1998, p. 113.)

42. Ibid., p. 116-117.

43. La question des références réelles au sein de la fiction se pose donc de façon relativement différente en fonction du médium par lequel la fiction est transmise : les théoriciens du cinéma s'interrogent ainsi depuis longtemps sur le rôle du corps réel des acteurs, et du décor réel, en dehors des personnages et des lieux fictionnels qu'ils représentent. Ce partage-là n'existe pas pour le texte de fiction.

44. Umberto Eco, op. cit., p. 117. 
Le genre intervient évidemment dans la définition de ce lecteur modèle : «L'œuvre de Dumas [se présente] stylistiquement et narrativement comme un roman historique populaire ${ }^{45}$. » Le lecteur de ce type de roman peut être gêné par un anachronisme important (qui pourrait, au pire, remettre en cause la vraisemblance du récit) mais les informations historiques demeurent pour lui à l'arrière-plan de la fiction.

\section{Pseudo-érudition, légende, utopie, méta-fiction... Chaque ouvrage présente un pacte spécifique}

Le texte d'Audeguy joue quant à lui sur les références à des personnages historiques en mêlant le vrai et le faux. Dans des entretiens, l'auteur a ainsi confié qu'il a remanié certaines biographies, par exemple celle d'Abercrombie, qui n'a jamais écrit le protocole que le roman lui prête, et en a inventé d'autres, ainsi du peintre Carmichael, inspiré librement de Constable, alors qu'il est resté fidèle à la vie de Luke Howard ${ }^{46}$. Sa pratique se situe dans la lignée de la pseudo-érudition perecquienne, qui engendre des textes trompeurs, que Perec rapproche des trompe-l'œil ${ }^{47}$. Pour prendre l'exemple du « romans » La Vie mode d'emploi, il se permet d'inventer en ce qui concerne l'arrière-plan de la fiction, a priori réel dans cette fiction pseudo-réaliste, arrière-plan de surcroît hypertrophié. On est le plus souvent au-delà de l'encyclopédie possible du lecteur, même érudit, qui est donc réduit à faire confiance au texte, alors même que des erreurs évidentes apparaissent et que divers indices sèment le doute. Le lecteur oscille entre plusieurs attitudes à l'égard du texte, ou se trouve maintenu dans une incertitude.

Audeguy lui aussi mêle vrai et faux mais il ne cherche pas à tromper son lecteur, ni à le faire douter. L'épitexte confirme la piste de lecture fournie par le texte : le pacte perçu par Virginie Latour, qui est pour elle un pacte biofictionnel, devient pour le lecteur une piste de lecture concernant les

45. Ibid., p. 116. Searle a déjà fait observer que le genre détermine le « degré de réalité » présent au sein de la fiction : «La différence entre les romans naturalistes, les contes de fées, les ouvrages de science-fiction et les récits surréalistes est en partie fonction du degré auquel l'auteur se met en devoir de représenter des faits réels, que ce soient des faits précis sur des lieux déterminés, comme Londres, Dublin ou la Russie, ou des faits généraux sur le comportement probable des gens ou sur la marche du monde. » (Op. cit., p. 116-117.) Le genre détermine également l'enjeu des références réelles pour le lecteur : l'importance qu'il leur accorde et la manière dont il les interprète.

46. Voir Shadi Biglarzadeh, «Interview de Stéphane Audeguy », sur le site @lalettre.com, http://www.alalettre.com/interview-stephane-audeguy.php (consulté le 15 juin 2011). Voir aussi Alexandra Morardet, «Stéphane Audeguy, écrivain » (entretien), sur le site arte.tv, http://www.arte.tv/fr/1085522,CmC=1085570.html (consulté le 15 juin 2011).

47. Voir mon article «Le trompe-l'œil, image usée d'un usage perecquien de la fiction» (version mise à jour de cette intervention), colloque Frontières de la fiction, 1999, [En ligne], http://www.fabula.org/colloques/frontieres/367.php (consulté le 15 juin 2011). 
personnages secondaires, objets de récits dans le récit. Ceux-ci ne relèvent plus de la question du vrai ou du faux : les éléments prélevés dans la réalité, repris dans des ensembles indécidables, «se transforment en élément de fiction », selon la conception de Genette. Alors qu'il s'agit de faits qui dépassent son encyclopédie ${ }^{48}$, le lecteur doit donc renoncer à voir dans le livre une source d'informations : l'indication d'un domaine, tout au plus.

Audeguy a déclaré qu'il recherchait davantage l'exactitude que la vérité ${ }^{49}$. Il prête dans son roman cette préoccupation à Akira Kumo, nous l'avons vu : l'exactitude semble correspondre à cette " vérité supérieure à celle des faits objectivement constatables » que rechercherait le personnage. On peut donc émettre l'hypothèse qu'Audeguy chercherait dans son roman à retracer (ou à approcher) l'essence, ou l'essentiel, de la réalité. Cela correspondrait à la manière schématique dont les personnages sont caractérisés ${ }^{50}$. Si l'histoire d'Akira Kumo est plus développée que celle de Virginie Latour, dont on ne connaît pas le passé, elle est emblématique de notre époque, s'originant dans la catastrophe d'Hiroshima. Elle correspond à la volonté de traiter de « la violence scientifique et la violence "occidentale" 51 ", et de bâtir un légendaire pleinement contemporain.

L'enjeu de la confrontation entre réalité et fiction élaborée par Ourania est assez différent. C'est le genre utopique qui est repris ${ }^{52}$, avec un décalage correspondant à sa transposition dans le présent. L' « étroite filiation » avec le texte fondateur de More a déjà été notée ${ }^{53}$.

L'idée même d'utopie repose sur une pluralité d'ambiguïtés. Au plan conceptuel tout d'abord : l'utopie forme tantôt l'esquisse d'une société idéale - reflet possible d'une doctrine sociopolitique - et tantôt la dénonciation d'un monde aliéné d'où naît l'espoir d'un système meilleur - à l'horizon de l'ambition réformiste. Moralement positivée, l'utopie affirme la volonté d'un dépassement nécessaire du présent; mais elle nous projette

48. Audeguy confie dans un entretien : «La documentation pour le roman était énorme. » Même s'il n'en a utilisé que « $20 \%$ », on se situe au-delà de l'encyclopédie spontanée d'un lecteur (Alexandra Morardet, op. cit.).

49. «J'espère que tout est le plus exact possible, ce qui est pour moi plus important que l'opposition vrai-faux...» (entretien avec Shadi Biglarzadeh, op. cit.).

50. Ce schématisme peut correspondre à un refus de l'explication psychologique. Il est dit d'un des personnages : «Il n'aime pas la psychologie. Il pense qu'il ne faut pas expliquer les gens, jamais, qu'on ne peut pas faire le tour d'un homme comme on fait celui d'un monument, qu'il faut laisser les gens tranquilles, même quand ils sont morts. » (Stéphane Audeguy, op. cit., p. 247.) Le livre s'appuie aussi sur des stéréotypes, ce qui a été remarqué à propos du personnage de Virginie Latour. (Voir l'entretien avec Alexandra Morardet, $o p$. cit.) Comme le Japonais est couturier, la bibliothécaire est bornée, l'intellectuelle naïve.

51. Entretien avec Biglarzadeh, op. cit.

52. Voir entretien Jérôme Garcin, « Voyage en utopie, un entretien avec J.M.G. Le Clézio », Le Nouvel Observateur, 2 février 2006, p. 87-88. Voir aussi, J.-M. G. Le Clézio, op. cit., p. 76.

53. Claude Cavallero, op. cit., p. 216. 
aussitôt dans la sphère du rêve, de l'imaginaire, ce qui relègue au second plan l'action politique concrète.

Le roman de Le Clézio se trouve largement affecté par de telles ambivalences, et c'est pourquoi se pose avec acuité la question de son interprétation ${ }^{54}$.

Le Clézio cherche peut-être, davantage, à dessiner la place possible des utopies dans notre monde actuel. Elles valent moins comme réalisations que comme fictions, pour les rêves qu'elles suscitent encore, malgré leurs échecs réels. Dès lors, le réalisme de Le Clézio, l'importance qu'il accorde à l'arrière-plan réel, avec le tableau socioéconomique précis du Mexique mondialisé retracé par le géographe Sillitoe, s'accorde avec la place de l'irréel, et en particulier, comme je l'ai dit, du rêve.

Dans La Vérité sur Marie, c'est l'arbitraire fictionnel qui est revendiqué, sur fond de vraisemblance, essentiellement psychologique. L'arrière-plan géographique est peu dessiné, seuls quelques lieux précis émergent : l'appartement de la rue de La Vrillière, l'aéroport de Narita, la maison de l'île d'Elbe... L'île d'Elbe n'est d'ailleurs pas caractérisée précisément. Elle est avant tout une île méditerranéenne et pour la décrire, l'auteur a confié s'être inspiré de sa connaissance de la Corse ${ }^{55}$. Les bévues référentielles sont rares mais le récit n'hésite pas à les souligner, comme il laisse apparaitre, je l'ai dit, des incohérences marginales. Ainsi, au sein d'une scène de décollage très détaillée et informée ${ }^{56}$, le narrateur affirme qu'un cheval vomit tout en remarquant que c'est physiquement impossible. Pourquoi ne corrige-t-il pas? Certainement parce que l'erreur est préférable à la réalité, l'imaginaire et le rêve au réel.

\footnotetext{
Car Zahir était autant dans la réalité que dans l'imaginaire, dans cet avion en vol que dans les brumes d'une conscience, ou d'un rêve, inconnu, sombre, agité, où les turbulences du ciel sont des fulgurances de la langue, et, si, dans la réalité, les chevaux ne vomissent pas, ne peuvent pas vomir (il leur est physiquement impossible de vomir, leur organisme ne le leur permet pas, même quand ils ont mal au cœur, même quand leur estomac est surchargé de substances toxiques), Zahir, [...] cette nuit, indifférent à sa nature, traître à son espèce, se mit à vomir dans le ciel dans les soutes du Boeing 747 cargo qui volait dans la nuit ${ }^{57}$.
}

54. Ibid., p. 209-210.

55. Voir Marie Desplechin, « Jean-Philippe Toussaint : “Je cherche une énergie romanesque pure” », Le Monde, 18 septembre 2009 : «l'île d'Elbe, c'est la Corse. Mais l'île d'Elbe en même temps. Pour preuve : il a les plans » (p. 8).

56. Le site Jean-Philippe Toussaint donne accès à une correspondance de l'auteur avec Guilhem Perrichet, commandant de bord, auprès duquel il s'est renseigné très précisément sur les aspects techniques et les perceptions lors du décollage à bord d'un Boeing 747, « Correspondance avec Guilhem Perrichet, commandant de bord », http://www.jptoussaint. com/documents/0/0e/Correspondance_avec_Guilhem_Perricher\%2C_commandant_de bord_Air_France.pdf (consulté le 15 septembre 2011).

57. Jean-Philippe Toussaint, op. cit., p. 137-138. Sur ce passage, voici ce que l'auteur a confié à Laurent Demoulin, op. cit. : «La première image que j'ai eue de ce livre, c'est un cheval qui vomit dans la soute d'un Boeing 747 en vol. J'ai construit le roman pour arriver 
Pour reprendre les termes du narrateur déjà cités, la « vérité nouvelle » qui est visée ne se soucie pas « de vraisemblance ou de véracité », elle est « jumelle du mensonge ». Le lecteur, qui aurait pu ne pas remarquer l'erreur, doit admettre l'invraisemblance du récit qui reste, je l'ai dit, marginale, sur fond de vraisemblance. Ainsi, la relation au référent est problématisée, et la fiction joue avec ses présupposés ${ }^{58}$. Le caractère contrefactuel du récit se trouve donc souligné. La fiction, joueuse, inconséquente, s'assume comme telle. D'où les longs passages à portée métatextuelle. Et si la fiction est présentée comme supérieure à la réalité, source d'une « vérité idéale », c'est comme le remarque Frank Wagner, que se trouve disqualifiée « toute prétention à quelque vérité objective que ce $\operatorname{soit}^{59}{ }^{59}$.

\section{Conclusion}

On a raison d'opposer pacte fictionnel et pacte référentiel. Il faut remarquer cependant qu'au sein du fictionnel, la part laissée à la fiction peut être plus ou moins grande : la fiction est plus ou moins tenue par la vraisemblance, et surtout la quantité de faits réels insérés dans la diégèse est plus ou moins importante. Enfin, l'enjeu du récit peut plus ou moins résider dans cette part fictionnelle. Finalement, chaque roman détermine un pacte spécifique, en particulier par son inscription générique.

Les possibilités sont très diverses, comme le montrent les trois exemples étudiés ici, d'autant que rien n'empêche que le pacte, plus ou moins implicite, se révèle trompeur. Ourania souligne l'écart entre un réel décevant et un rêve déçu. La désignation d'une confusion des deux, à la fin de l'ouvrage, désigne l'espoir maintenu que l'un puisse contaminer l'autre, l'affirmation que l'utopie peut exister. La Théorie des nuages cherche à tracer de grandes lignes, à schématiser l'histoire, tout en interrogeant le

à cette scène-là. Car la force poétique de cette image me plaisait. Il s'est trouvé que, dès que j'ai commencé à travailler, je me suis informé sur les chevaux et j'ai appris qu'ils ne vomissaient pas. Ça commençait mal... Cela m'a amené à prendre une position narrative très radicale : j'affirme quelque chose d'impossible. Pourtant, je ne sacrifie pas du tout le réalisme. J'essaie de rendre l'avion le plus réaliste possible, que l'on y soit vraiment : je décris des sensations, des vibrations, des détails pour produire un effet de réel. Le cheval est là. Et, bien que dans la réalité les chevaux ne puissent pas vomir, il vomit. D'une certaine façon, je romps le pacte tacite entre le lecteur et moi, qui voudrait que je raconte des choses plutôt vraisemblables. Après avoir fait croire à la réalité de la description, j'affirme soudain que l'on n'est pas dans un avion en vol mais dans la littérature. »

58. C'est à l'époque de La Salle de bain la fantaisie qui, davantage, régulait «l'imagination romanesque en la renvoyant au souvenir de son arbitraire » (Bruno Blanckeman, Les Fictions singulières, étude sur le roman français contemporain, Paris, Prétexte, coll. « Critique », 2002, p. 69).

59. Frank Wagner, «La vérité sur Jean-Philippe (éléments pour une poétique de l'œuvre toussainienne », Vox-poetica, [En ligne], http://www.vox-poetica.org/t/articles/wagner2011. html (consulté le 15 septembre 2011). 
mystère des passions humaines. Il intègre le savoir à la fiction, à tel point que son lecteur modèle doit se comporter comme Genette, et ne plus interroger la vérité des références à des personnages historiques. La Vérité sur Marie est un titre ironique : elle n'existe pas. C'est la fiction que le roman exalte, suggérant ses infinis pouvoirs à travers quelques scènes, dont le fil lâche est une histoire d'amour au dénouement attendu, ou la poursuite d'un(e) personnage énigmatique ${ }^{60}$. Comme l'a fait remarquer Frank Wagner, la « vérité idéale », « jumelle du mensonge » qu'exalte le narrateur n'est autre que la vérité fictionnelle : une vérité autre ${ }^{61}$. La réalité n'est pas la fiction. Tout lecteur sait parfaitement faire la différence. La fiction peut pourtant jouer de ce partage, ou prétendre le faire. Elle peut élaborer des fictions à partir d'éléments réels, elle peut confronter réalité et imaginaire, elle peut schématiser la réalité, s'en éloigner ou encore mettre en doute ces opérations.

Les trois romans contemporains que j'ai étudiés s'attachent à la fiction qui les constitue, en des passages métatextuels qui conduisent leur lecteur à s'interroger sur ses pouvoirs. Ce n'est pas absolument nouveau, puisque de telles interrogations s'observent dès les origines du roman. Il est intéressant d'observer qu'aujourd'hui, l'accent est mis par plusieurs romans sur une limite qu'interroge justement la théorie de la fiction actuelle : celle des références réelles au sein de la fiction. En tout cas, ce retour de la fiction sur elle-même est caractéristique du roman de notre époque, d'un intérêt porté à la fiction en soi, d'une conscience de ses fonctionnements, d'une volonté d'en explorer les limites.

Cécile De Bary Université Paris Diderot, Sorbonne Paris Cité, Cerilac

60. Bruno Blanckeman a montré comment dès Faire l'amour, la structure narrative passe au second plan derrière une suite de scènes qui constituent autant de «points de tension »: « À [la] défection des figures romanesques, qui ne vaut pas pour abandon de la figuration, répond un défaut de structure narrative, qui n'équivaut pas à un manque de structuration. » ( "Faire l'amour à la Toussaint», dans Marc Dambre et Bruno Blanckeman (dir.), Romanciers minimalistes, 1979-2003, Paris, Presses Sorbonne nouvelle, 2012, p. 151.)

61. Ibid. 